hep-ph/9810471

DPNU-98-36

KEK-TH-597

OHSTPY-HEP-T-98-022

Oct. 1998

\title{
The renormalization group analysis of the large lepton flavor mixing and the neutrino mass
}

\author{
N. Haba ${ }^{1} * \quad$ N. Okamura ${ }^{2}+\quad$ M. Sugiura ${ }^{3}$ S $^{-}$ \\ ${ }^{1}$ Department of Physics, The Ohio State University, Columbus, Ohio 43210, USA \\ ${ }^{2}$ Theory Group, KEK, Oho 1-1, Tsukuba 305-0801, Japan \\ ${ }^{3}$ Department of Physics, Nagoya University, Nagoya, 464-8602, Japan
}

\begin{abstract}
The Superkamiokande experiment suggests the large flavor mixing between $\nu_{\mu}$ and $\nu_{\tau}$. We show that the mixing angle receives significant corrections from the renormalization group equation (RGE) when both the second and the third generation neutrino masses are larger than $O(0.1 \mathrm{eV})$. This means that the mixing angle must be small at the decoupling scale of right-handed neutrinos in the model containing a sterile neutrino $\nu_{s}$ with the mass spectrum of $m_{\nu_{s}} \approx m_{\nu_{e}} \ll m_{\nu_{\mu}} \approx m_{\nu_{\tau}}$.
\end{abstract}

\footnotetext{
${ }^{*}$ E-mail: haba@pacific.mps.ohio-state.edu

$\dagger$ E-mail: okamura@theory.kek.jp

${ }_{\ddagger}^{\ddagger}$ E-mail: sugiura@eken.phys.nagoya-u.ac.jp
} 


\section{Introduction}

The recent Superkamiokande data suggests the large neutrino flavor mixing between $\nu_{\mu}$ and $\nu_{\tau}[1$. According to this experimental result, there have been a lot of theoretical attempts to explain why the large flavor mixing is realized in the lepton sector [2]. One of the interesting approaches is concentrating on the effects of the the renormalization group equation (RGE). The RGE effects cause the enhancement of the neutrino flavor mixing in some situations [3] [4] [5].

In this paper we analyze the RGE of the neutrino flavor mixing between $\nu_{\mu}$ and $\nu_{\tau}$ in the minimal supersymmetric standard model (MSSM) with right-handed neutrinos. Here we stand the position that the smallness of neutrino masses is explained by the seesaw mechanism[6]. We consider the situation that $m_{\nu_{e}}$ is much smaller than $m_{\nu_{\mu}}$ and $m_{\nu_{\tau}}$, and expect the solar neutrino problem[7] is solved by the oscillation between $\nu_{e}$ and a sterile neutrino $\nu_{s}[$ 8]. This situation is so-called four light neutrino scenario [9] [10] with the mass spectrum of $m_{\nu_{s}} \approx m_{\nu_{e}} \ll m_{\nu_{\mu}} \approx m_{\nu_{\tau}}$. In this neutrino mass hierarchy, we find that the mixing angle between $\nu_{\mu}$ and $\nu_{\tau}$ receives significant corrections from the renormalization group equation (RGE). In this case the mixing angle at the high energy must be small as long as the mixing at the low energy is maximal. From the view point of the model building, we must find the fundamental theory which induces the small mixing angle at the high energy in the four neutrino scenario with the above mass spectrum.

\section{The RGE effects of the neutrino flavor mixing}

\footnotetext{
$\S$ The four neutrino scenario might also explain the results of the LSND 11]. The LSND results suggest the small mixing between $\bar{\nu}_{\mu}$ and $\bar{\nu}_{e}$ with $m_{\nu_{\mu}}^{2}-m_{\nu_{e}}^{2} \sim 1 \mathrm{eV}$. However, the confirmation of this result still awaits future experiments. Recent measurements in the KARMEN detector exclude part of the LSND allowed region[12].
} 


\subsection{The RGEs of the Yukawa couplings}

In this section we show the RGEs of the MSSM with right-handed neutrinos. The superpotential of the MSSM is given by

$\mathcal{W}=y_{i j}^{\mathrm{u}}{ }_{i j} Q_{i} H_{\mathrm{u}} \bar{U}_{j}+y^{\mathrm{d}}{ }_{i j} Q_{i} H_{\mathrm{d}} \bar{D}_{j}+y^{\nu}{ }_{i j} L_{i} H_{\mathrm{u}} \bar{N}_{j}+y^{\mathrm{e}}{ }_{i j} L_{i} H_{\mathrm{d}} \bar{E}_{j}+\mu_{H} H_{\mathrm{u}} H_{\mathrm{d}}+\frac{1}{2} M_{R i j} \bar{N}_{i} \bar{N}_{j}$,

where the index $i, j$ stands for the generation number $(i, j=2,3)$. In this paper we neglect Yukawa couplings of the first generation since we consider the case where $m_{\nu_{e}}$ is much smaller than $m_{\nu_{\mu}}$ and $m_{\nu_{\tau}} . Q_{i}, L_{i}, \bar{U}_{i}, \bar{D}_{i}, \bar{E}_{i}, \bar{N}_{i}$ and $H_{\mathrm{u}, \mathrm{d}}$ are quark doublet, lepton doublet, right-handed up-sector, right-handed down-sector, right-handed charged lepton, right-handed neutrino and Higgs fields, respectively. $M_{R}$ ij is the Majorana mass matrix of the right-handed neutrinos, which is symmetric under the generation indices $i, j . \mu_{H}$ is the supersymmetric mass parameter of Higgs particles.

In this model the RGEs of Yukawa couplings are given by

$$
\begin{aligned}
\frac{d}{d t} y^{\mathrm{u}} & =\frac{1}{(4 \pi)^{2}}\left[\operatorname{tr}\left(3 y^{\mathrm{u}} y^{\mathrm{u} \dagger}+y^{\nu} y^{\nu \dagger}\right)+3 y^{\mathrm{u}} y^{\mathrm{u} \dagger}+y^{\mathrm{d}} y^{\mathrm{d}^{\dagger}}-4 \pi\left(\frac{16}{3} \alpha_{3}+3 \alpha_{2}+\frac{13}{15} \alpha_{1}\right)\right] y^{\mathrm{u}}, \\
\frac{d}{d t} y^{\mathrm{d}} & =\frac{1}{(4 \pi)^{2}}\left[\operatorname{tr}\left(3 y^{\mathrm{d}} y^{\mathrm{d}^{\dagger}}+y^{\mathrm{e}} y^{\mathrm{e}^{\dagger}}\right)+3 y^{\mathrm{d}} y^{\mathrm{d}^{\dagger}}+y^{\mathrm{u}} y^{\mathrm{u} \dagger}-4 \pi\left(\frac{16}{3} \alpha_{3}+3 \alpha_{2}+\frac{7}{15} \alpha_{1}\right)\right] y^{\mathrm{d}}, \\
\frac{d}{d t} y^{\mathrm{e}} & =\frac{1}{(4 \pi)^{2}}\left[\operatorname{tr}\left(3 y^{\mathrm{d}} y^{\mathrm{d}^{\dagger}}+y^{\mathrm{e}} y^{\mathrm{e} \dagger}\right)+3 y^{\mathrm{e}} y^{\mathrm{e}^{\dagger}}+y^{\nu} y^{\nu \dagger}-4 \pi\left(3 \alpha_{2}+\frac{9}{5} \alpha_{1}\right)\right] y^{\mathrm{e}}, \\
\frac{d}{d t} y^{\nu} & =\frac{1}{(4 \pi)^{2}}\left[\operatorname{tr}\left(3 y^{\mathrm{u}} y^{\mathrm{u} \dagger}+y^{\nu} y^{\nu \dagger}\right)+3 y^{\nu} y^{\nu \dagger}+y^{\mathrm{e}} y^{\mathrm{e} \dagger}-4 \pi\left(3 \alpha_{2}+\frac{3}{5} \alpha_{1}\right)\right] y^{\nu},
\end{aligned}
$$

where $t=\ln \mu$ and $\mu$ is a renormalization point, respectively. These equations are available for the energy region of $\mu>\mathcal{M}_{\mathcal{R}}$, where $\mathcal{M}_{\mathcal{R}}$ denotes the energy scale of the Majorana mass.

Below the scale of $\mathcal{M}_{\mathcal{R}}$, we should take the decoupling effects of heavy neutrinos into account. The effective theory is described without heavy neutrinos. The 
superpotential of Eq.(11) is modified as

$$
\mathcal{W}=y^{\mathrm{u}}{ }_{i j} Q_{i} H_{\mathrm{u}} \bar{U}_{j}+y^{\mathrm{d}}{ }_{i j} Q_{i} H_{\mathrm{d}} \bar{D}_{j}+y^{\mathrm{e}}{ }_{i j} L_{i} H_{\mathrm{d}} \bar{E}_{j}-\frac{1}{2} \kappa_{i j} \nu_{i} \nu_{j} H_{\mathrm{u}} H_{\mathrm{u}} .
$$

Here $\nu_{i}$ s are the light modes of neutrinos which remain after integrating out the heavy ones. The coupling constant $\kappa_{i j}$ is defined as

$$
\kappa_{i j}=\left(y^{\nu} M_{R}^{-1} y^{\nu T}\right)_{i j} .
$$

It relates to the mass matrix of the light neutrinos as

$$
m_{i j}^{\nu}=\frac{v_{\mathrm{u}}^{2}}{2} \kappa_{i j}=\frac{v^{2} \sin ^{2} \beta}{2} \kappa_{i j}
$$

where

$$
\tan \beta \equiv \frac{v_{\mathrm{u}}}{v_{\mathrm{d}}}, \quad v^{2}=v_{\mathrm{u}}^{2}+v_{\mathrm{d}}^{2}
$$

with $\left\langle H_{\mathrm{u}}\right\rangle=v_{\mathrm{u}}$ and $\left\langle H_{\mathrm{d}}\right\rangle=v_{\mathrm{d}}$. The value of $v$ is given by

$$
v=M_{Z} \frac{\sin 2 \theta_{W}}{2} \sqrt{\frac{\alpha}{\pi}}=245.4(\mathrm{GeV})
$$

with $M_{Z}=91.187 \mathrm{GeV}, \alpha=127.9$, and $\sin ^{2} \theta_{W}=0.2313$.

In $\mu<\mathcal{M}_{\mathcal{R}}$, the RGEs of the Yukawa couplings Eqs.(2) are modified as

$$
\begin{aligned}
\frac{d}{d t} y^{\mathrm{u}} & =\frac{1}{(4 \pi)^{2}}\left[\operatorname{tr}\left(3 y^{\mathrm{u}} y^{\mathrm{u} \dagger}\right)+3 y^{\mathrm{u}} y^{\mathrm{u} \dagger}+y^{\mathrm{d}} y^{\mathrm{d}^{\dagger}}-4 \pi\left(\frac{16}{3} \alpha_{3}+3 \alpha_{2}+\frac{13}{15} \alpha_{1}\right)\right] y^{\mathrm{u}}, \\
\frac{d}{d t} y^{\mathrm{d}} & =\frac{1}{(4 \pi)^{2}}\left[\operatorname{tr}\left(3 y^{\mathrm{d}} y^{\mathrm{d}^{\dagger}}+y^{\mathrm{e}} y^{\mathrm{e} \dagger}\right)+3 y^{\mathrm{d}} y^{\mathrm{d}^{\dagger}}+y^{\mathrm{u}} y^{\mathrm{u} \dagger}-4 \pi\left(\frac{16}{3} \alpha_{3}+3 \alpha_{2}+\frac{7}{15} \alpha_{1}\right)\right] y^{\mathrm{d}}, \\
\frac{d}{d t} y^{\mathrm{e}} & =\frac{1}{(4 \pi)^{2}}\left[\operatorname{tr}\left(3 y^{\mathrm{d}} y^{\mathrm{d}^{\dagger}}+y^{\mathrm{e}} y^{\mathrm{e} \dagger}\right)+3 y^{\mathrm{e}} y^{\mathrm{e} \dagger}-4 \pi\left(3 \alpha_{2}+\frac{9}{5} \alpha_{1}\right)\right] y^{\mathrm{e}},
\end{aligned}
$$

and

$$
\frac{d}{d t} \kappa=\frac{1}{8 \pi^{2}}\left[\left\{\operatorname{tr}\left(3 y^{\mathrm{u}} y^{\mathrm{u} \dagger}\right)-4 \pi\left(3 \alpha_{2}+\frac{3}{5} \alpha_{1}\right)\right\} \kappa+\frac{1}{2}\left\{\left(y^{\mathrm{e}} y^{\mathrm{e} \dagger}\right) \kappa+\kappa\left(y^{\mathrm{e}} y^{\mathrm{e} \dagger}\right)^{T}\right\}\right] .
$$

From Eqs.(8), we can see that the RGEs of quark and charged lepton do not include the neutrino Yukawa couplings contrary to the case of $\mu>\mathcal{M}_{\mathcal{R}}$ as Eqs.(2). Hence below $\mathcal{M}_{\mathcal{R}}$ the running of the Yukawa couplings of quark and charged lepton can be determined independently of that of the neutrinos. 


\subsection{The RGEs of neutrinos in the effective theory}

From now on we are concentrating on the RGE effects below the scale of $\mathcal{M}_{\mathcal{R}}$, which are given by Eqs.(8) and (91). Since RGEs of the Yukawa couplings for quark and charged lepton of Eqs. (8) can be solved without information about the neutrino sector as mentioned before, the renormalization point dependences of $y_{\mu}$ and $y_{\tau}$ are completely determined by the RGEs' boundary conditions which we take the masses of quark and charged lepton 9 , the Cabibbo-Kobayashi-Maskawa matrix [14] and $\tan \beta$ at the weak scale. Then we have only to concentrate on Eq.(91). Here we neglect $C P$ phases in the flavor mixing matrices of the quark and the lepton sector for simplicity.

For convenience, we take three independent parameters $\kappa_{r} \equiv \kappa_{22} / \kappa_{33}, \sin ^{2} 2 \theta_{23}$ and $\delta \kappa^{2}$ instead of $\kappa_{i j}\left[\kappa_{22}, \kappa_{33}\right.$ and $\left.\kappa_{23}\left(=\kappa_{32}\right)\right]$. Here $\sin \theta_{23}$ and $\delta \kappa^{2}$ are determined from $\kappa_{i j}$ by the following equations:

$$
\begin{aligned}
\kappa & =\left(\begin{array}{cc}
\cos \theta_{23} & \sin \theta_{23} \\
-\sin \theta_{23} & \cos \theta_{23}
\end{array}\right)\left(\begin{array}{cc}
\kappa_{2} & 0 \\
0 & \kappa_{3}
\end{array}\right)\left(\begin{array}{cc}
\cos \theta_{23} & -\sin \theta_{23} \\
\sin \theta_{23} & \cos \theta_{23}
\end{array}\right), \\
\delta \kappa^{2} & \equiv \kappa_{3}^{2}-\kappa_{2}^{2}
\end{aligned}
$$

where

$$
\kappa_{3}=\frac{\sqrt{\delta \kappa^{2}}}{2}\left(\sqrt{\alpha}+\frac{1}{\sqrt{\alpha}}\right), \quad \kappa_{2}=\frac{\sqrt{\delta \kappa^{2}}}{2}\left(\sqrt{\alpha}-\frac{1}{\sqrt{\alpha}}\right)
$$

with

$$
\alpha \equiv\left|\frac{1+\kappa_{r}}{1-\kappa_{r}} \cos 2 \theta_{23}\right| .
$$

By using this relation, the RGE of Eq.(9) can be rewritten into the following three equations

$$
\frac{d}{d t} \kappa_{r}=-\frac{1}{8 \pi^{2}}\left(y_{\tau}^{2}-y_{\mu}^{2}\right) \kappa_{r}
$$

I We use $m_{t}=174.5 \mathrm{GeV}, m_{c}=0.657 \mathrm{GeV}, m_{b}=3.02 \mathrm{GeV}, m_{s}=9.935 \times 10^{-2} \mathrm{GeV}, m_{\tau}=1.746$ $\mathrm{GeV}$, and $m_{\mu}=1.0273 \times 10^{-3} \mathrm{GeV}$ at $\mu=M_{Z}[13]$.

I| Equation (15) was firstly derived by Babu, Leung and Pantaleone in Ref. [3]. 


$$
\begin{aligned}
\frac{d}{d t} \sin ^{2} 2 \theta_{23}= & -\frac{1}{8 \pi^{2}} \sin ^{2} 2 \theta_{23}\left(1-\sin ^{2} 2 \theta_{23}\right)\left(y_{\tau}^{2}-y_{\mu}^{2}\right) \frac{1+\kappa_{r}}{1-\kappa_{r}} \\
\frac{d}{d t} \delta \kappa^{2}= & \frac{1}{8 \pi^{2}}\left[2\left\{\operatorname{tr}\left(3 y^{\mathrm{u}} y^{\mathrm{u} \dagger}\right)-4 \pi\left(3 \alpha_{2}+\frac{3}{5} \alpha_{1}\right)\right\}+y_{\tau}^{2}+y_{\mu}^{2}\right. \\
& \left.+\left(y_{\tau}^{2}-y_{\mu}^{2}\right)\left(\frac{1+\kappa_{r}^{2}}{1-\kappa_{r}^{2}}-\frac{1}{2} \cdot \frac{1+\kappa_{r}}{1-\kappa_{r}} \sin ^{2} 2 \theta_{23}\right)\right] \delta \kappa^{2}
\end{aligned}
$$

Both $\sin ^{2} 2 \theta_{23}$ and $\delta \kappa^{2}$ directly relate to the observed quantities in neutrino oscillation experiment:**. The mass squared difference can be written as $\delta m^{2}=v^{4} \sin ^{4} \beta \delta \kappa^{2} / 4$ by using Eq.(15).

As shown before, $y_{\mu}(\mu)$ and $y_{\tau}(\mu)$ are determined without knowing the neutrino Yukawa coupling, thus we can obtain values of $\sin 2 \theta_{23}$ and $\kappa_{r}$ at the weak scale by using only Eqs.(14) and (15). We analyze RGEs of Eqs.(14) and (15) by inputting various values of $\sin \theta_{23}$ and $\kappa_{r}$ for the initial conditions at $\mu=\mathcal{M}_{\mathcal{R}}{ }^{\dagger \dagger}$.

\subsection{Numerical results of the RGEs}

Now we show the numerical results of the RGEs. Figure 1 shows the energy dependence of the values of $\left[y_{\tau}^{2}-y_{\mu}^{2}\right]$, which is the coefficients of the RGEs of $\kappa_{r}$ in Eq.(14) and $\sin ^{2} 2 \theta_{23}$ in Eq.(15). Four lines correspond to the various values of $\tan \beta$, which we take $5,20,35$, and 50 . The values of $\left[y_{\tau}^{2}-y_{\mu}^{2}\right]$ do not receive the significant RGE corrections when $\tan \beta$ is small.

Figures 2 show the energy dependence of $\kappa_{r}$ with $\tan \beta=50$. We show the two cases of (a): $\mathcal{M}_{\mathcal{R}}=10^{14} \mathrm{GeV}$ and (b):10 ${ }^{16} \mathrm{GeV}$ with various initial conditions at $\mathcal{M}_{\mathcal{R}}$. From the high energy to the low energy, $\kappa_{r}$ simply increases. If we take the small $\tan \beta$, the slope tends to be flat because the value of $\left[y_{\tau}^{2}-y_{\mu}^{2}\right]$ in Eq.(14) decreases as Fig. 11]

\footnotetext{
${ }^{* *}$ Since we take a diagonal base of the charged lepton, $\sin ^{2} 2 \theta_{23}$ of this paper is an observable quantity.

${ }^{\dagger \dagger}$ As shown later, we do not need to calculate Eq.(16) in our analysis.
} 
Figures 3 show the energy dependence of the mixing angle with the same values of $\mathcal{M}_{\mathcal{R}}$ and $\kappa_{r}\left(\mathcal{M}_{\mathcal{R}}\right)$ as Figs. 2] There are three curves corresponding to the values of $\kappa_{r}\left(\mathcal{M}_{\mathcal{R}}\right)$, and all of them have the same boundary condition of $\sin ^{2} 2 \theta_{23}\left(\mathcal{M}_{\mathcal{R}}\right)=$ 0.1. From these figures we can see that the mixing angle at the weak scale changes depending on the value of $\kappa_{r}\left(\mathcal{M}_{\mathcal{R}}\right)$. This difference can be easily understood by comparing Figs. 3 with Figs. 2 and by the existence of the factor $\left[\left(1+\kappa_{r}\right) /\left(1-\kappa_{r}\right)\right]$ in the R.H.S. of the RGE of Eq.(15). Let us see Fig. 2(a) and Fig. 3(a), for example. In the case of $\kappa_{r}\left(\mathcal{M}_{\mathcal{R}}\right)=0.8$, where $\kappa_{r}$ does not exceed one at all the energy scales, the mixing angle does not receive the significant RGE corrections. It is because the R.H.S. of Eq.(15) does not become so large as to enhance the mixing angle. On the other hand, in the case of $\kappa_{r}\left(\mathcal{M}_{\mathcal{R}}\right)=0.9 \kappa_{r}$ exceeds one near the weak scale, which results in the significant enhancement of the mixing angle by the factor $\left[\left(1+\kappa_{r}\right) /\left(1-\kappa_{r}\right)\right]$. Finally in the case of $\kappa_{r}\left(\mathcal{M}_{\mathcal{R}}\right)=0.99, \kappa_{r}$ exceeds one above the weak scale. Then the mixing angle once becomes maximal at high energy, however, after there it decreases rapidly since the sign of $\left[\left(1+\kappa_{r}\right) /\left(1-\kappa_{r}\right)\right]$ changes.

Figures 4 are the contour plots of the mixing angle $\sin ^{2} 2 \theta_{23}$ at the weak scale. These are obtained by solving Eq.(15) with the various $\kappa_{r}\left(\mathcal{M}_{\mathcal{R}}\right)$ (horizontal axis) and $\sin ^{2} 2 \theta_{23}\left(\mathcal{M}_{\mathcal{R}}\right)$ (vertical axis). Here $\kappa_{c}$ in Figs. 4 is the value at $\mathcal{M}_{\mathcal{R}}$ that induces $\kappa_{r}($ weak $)=1$. In the parameter region of $\kappa_{r}\left(\mathcal{M}_{\mathcal{R}}\right)<\kappa_{c}$, there are no significant RGE corrections and then the mixing angle does not change drastically. The energy dependence of the mixing angle in this case is similar to the solid lines in Figs. 3 , Next in the case of $\kappa_{r}\left(\mathcal{M}_{\mathcal{R}}\right) \simeq \kappa_{c}$, which means $\kappa_{r}$ (weak) $\simeq 1$, the mixing angle at the weak scale is strongly enhanced near the weak scale, as the dashed lines in Figs. 3. Then the mixing angle at the weak scale becomes maximal independently of the mixing angle at $\mathcal{M}_{\mathcal{R}}$. Finally in the case of $\kappa_{r}\left(\mathcal{M}_{\mathcal{R}}\right)>\kappa_{c}$, the mixing angle at the weak scale becomes small even for the large mixing angle at $\mathcal{M}_{\mathcal{R}}$, where the 
energy dependence of the mixing angle is similar to the dotted lines in Figs. 3, From these arguments we can easily see that there is the large enhancement of the mixing angle from the RGE around $\kappa_{r} \simeq 1$.

Figures 5 are the contour plots of the heaviest neutrino mass $m_{3}$ at the weak scale. The horizontal and vertical axes are the same as those of Figs. 4, We determine the masses of neutrino by substituting the parameters in Eq.(12) with the result of Figs. (4) and the experimental value $\delta m_{23}^{2} \simeq 1.3 \times 10^{-3} \mathrm{eV}^{2}$. Since we use the experimental value of $\delta m_{23}^{2}$ instead of evaluating the RGE of Eq.(16), we can determine the masses without any additional input parameters. As $m_{3}$ becomes large, the region of $\kappa_{r}\left(\mathcal{M}_{\mathcal{R}}\right)$ is limited around $\kappa_{c}$. Compared with Figs. 4, it is found that the region where the heaviest mass $m_{3}$ is larger than $O(0.1 \mathrm{eV})$ corresponds to the region where the mixing angle at the weak scale is always larger than 0.9 despite the small mixing at $\mathcal{M}_{\mathcal{R}}$ scale.

Figures [6] and Figures 7 correspond to Figures 4 and Figures 5 with another value of $\tan \beta=35$. Compared with the case of $\tan \beta=50$, the value of $\kappa_{c}$ are just shifted to the right in the case of $\tan \beta=35$. Around $\kappa_{r}\left(\mathcal{M}_{\mathcal{R}}\right) \simeq \kappa_{c}$, the value of $m_{3}$ is slightly larger than that of $\tan \beta=50$ at the same value of $\sin ^{2} 2 \theta_{23}$. In general, the smaller $\tan \beta$ becomes, the more $\kappa_{c}$ approaches one, and the value of $m_{3}$ around $\kappa_{r}\left(\mathcal{M}_{\mathcal{R}}\right) \simeq \kappa_{c}$ becomes larger. The region where the maximal enhancement of the mixing angle is derived by the RGE exists even in the case of small $\tan \beta$. The value of $m_{3}$ around $\kappa_{c}$ becomes larger corresponding to the smaller value of $\tan \beta$.

We stress here that the large enhancement factor at the weak scale induced by $\left[\left(1+\kappa_{r}\right) /\left(1-\kappa_{r}\right)\right]$ in Eq.(15) is not the fine-tuning. This factor must be inevitably large with $\kappa_{r}($ weak $) \simeq 1$ if $m_{2}$ and $m_{3}$ are larger than $O(0.1 \mathrm{eV})$. It is worth noting that the enough enhancement of the mixing angle can be obtained even in the case of $O(0.1 \mathrm{eV})$ neutrino masses. Even in the small $\tan \beta, O(1 \mathrm{eV})$ mass is enough to obtain 
this RGE enhancement.

\section{Summary and Discussion}

In this paper we analyzed the RGE effects of the neutrino flavor mixing between $\nu_{\mu}$ and $\nu_{\tau}$ in the MSSM with right-handed neutrinos. The experimental result of the Superkamiokande suggests the large neutrino flavor mixing between $\nu_{\mu}$ and $\nu_{\tau}$ with $\delta m_{23}^{2} \simeq 10^{-3} \mathrm{eV}$. Then we found that the mixing angle between $\nu_{\mu}$ and $\nu_{\tau}$ receives significant corrections from the RGE in the case of $m_{\nu_{s}} \approx m_{\nu_{e}} \ll m_{\nu_{\mu}} \approx m_{\nu_{\tau}}$. In this mass spectrum, the mixing angle at the decoupling scale of right-handed neutrinos must be small when $O(0.1 \mathrm{eV}) \leq m_{\nu_{\tau}}\left(\approx m_{\nu_{\mu}}\right)$.

Finally we give a brief comment about the LSND experimental result. In the above mass spectrum, both $m_{\nu_{\mu}}$ and $m_{\nu_{\tau}}$ must be of order $1 \mathrm{eV}$ to explain the LSND result. In this case, the RGE analysis shows that the mixing angle at the decoupling scale of right-handed neutrinos must be negligibly small $\$$. From the view point of the model building, we must find the fundamental theory which induces the small mixing angle at the high energy scale in the four neutrino scenario with the mass spectrum of $m_{\nu_{s}} \approx m_{\nu_{e}} \ll m_{\nu_{\mu}} \approx m_{\nu_{\tau}}$.

\section{Acknowledgments}

We would like to thank M. Tanimoto for useful discussions. NO would like to thank K. Hagiwara, J. Hisano and Y. Okada for useful discussions and comments. NH would like to thank S. Raby and K. Tobe for helpful discussions and comments. The work of NO is financially supported by the JSPS Research Fellowships for young scientist, No.2996. NH is partially supposed by DOE grant DOE/ER/01545-753.

\footnotetext{
$\ddagger$ Although we have not shown the lines of $1 \mathrm{eV}$ in Figs. [5 and Figs. 7 there exist these lines. They are too small to be drawn apparently in the large $\tan \beta$.
} 


\section{References}

[1] Superkamiokande Collaboration, Y. Fukuda et al., Phys. Rev. Lett. 81 (1998) 1562; T. Kajita, talk at the International Conference Neutrino '98, Takayama, Japan, June 1998.

[2] See, for example, M. Fukugita, M. Tanimoto and T. Yanagida, Phys. Rev. D57 (1998) 4429; K.S. Babu and S.M. Barr, Phys. Lett. B381 (1996) 202; S.M. Barr, Phys. Rev. D55 (1997) 1659; B. Brahmachari and R.N. Mohapatra, Phys. Rev. D58 (1998) 15003; C.H. Albright and S.M. Barr, Phys. Rev. D58 (1998) 13002; J. Sato and T. Yanagida, Phys. Lett. B430 (1998) 127; S.L. Glashow, Phys. Lett. B256 (1991) 255; E.J. Chun, C.W. Kim and U.W. Lee, Phys. Rev. D58 (1998) 93003; Y. Chikira, N. Haba and Y. Mimura, hep-ph/9808254; E. Ma, hep-ph/9807386, M. Tanimoto, hep-ph/9807283, Y. Nomura and T. Yanagida, hep-ph/9807325 N. Haba, hep-ph/9807552 S. Davidson and S.F. King, hep-ph/9808296; R.N. Mohapatra and S. Nussinov, hep-ph/9808301; Y. Grossman, Y. Nir and Y. Shadmi, hep-ph/9808355 R.N. Mohapatra and S. Nussinov, hep-ph/9809415.

[3] K.S. Babu, C.N. Leung and J. Pantaleone, Phys. Lett. B319 (1993) 191.

[4] M. Tanimoto, Phys. Lett. B360 (1995) 41.

[5] N. Haba and T. Matsuoka, Prog. Theor. Phys. 99 (1998) 831; J. Ellis, G.K. Leontaris, S. Lola and D.V. Nanopoulos, hep-ph/9808251.

[6] T. Yanagida, in Proceedings of the Workshop on Unified Theory and Baryon Number in the Universe, ed. O. Sawada and A. Sugamoto (KEK, report 79-18, 1979), p.95; M. Gell-Mann, P. Ramond and S. Slansky, in Supergravity, ed. P. 
van Nieuwenhuizen and D. Z. Freedman (North-Holland, Amsterdam, 1979), p315; R. Mohapatra and S. Senjanović, Phys. Rev. Lett. 44 (1980) 912.

[7] GALLEX Collaboration, Phys. Lett. B388 (1996) 384; SAGE Collaboration, Phys. Lett. B328 (1994) 234; Homestake Collaboration, Nucl. Phys. B 38 (Proc. Suppl.) (1995) 47; Kamiokande Collaboration, Nucl. Phys. B 38 (Proc. Suppl.) (1995) 55; Super-Kamiokande Collaboration, Y. Fukuda et al., Phys. Rev. Lett. $81(1998) 1158$.

[8] See, for example, N. Hata and P. Langacker, Phys. Rev. D50 (1994) 632; ibid. D56 (1997) 6107; J.N. Bahcall, P.I. Krastev and A.Y. Smirnov, Phys. Rev. D58 (1998) 96016.

[9] See, for example, D.O. Caldwell, R.N. Mohapatra, Phys. Rev. D48 (1993) 3259; J.T. Peltoniemi, J.W.F. Valle, Nucl. Phys. B406 (1993) 409; R. Foot and R.R. Volkas, Phys. Rev D52 (1995) 6595; E. Ma and P. Roy, Phys. Rev. D52 (1995) 4780; J.J. Gomez-Cadenas and M.C. Gonzales-Garcia, Z. Phys. C71 (1996) 443; S.M. Bilenky, C. Giunti and W. Grimus, Proc. of Neutrino 96, Helsinki, June 1996, edited by K. Enqvist et. al., (World Scientific, 1997); S.M. Bilenky, C. Giunti and W. Grimus, Eur. Phys. J. C1 (1998) 247; N. Okada and O. Yasuda, Int. J. Mod. Phys. A12 (1997) 3669; S.C. Gibbons, R.N. Mohapatra, S. Nandi and A. Raychaudhuri, Phys. Lett. B430 (1998) 296; S.M. Bilenky, C. Giunti and W. Grimus, hep-ph/9805387; V. Barger, T.J. Weiler and K. Whisnant, Phys. Lett. B427 (1998) 97; V. Barger, S. Pakvasa, T.J. Weiler and K. Whisnant, Phys. Rev. D58 (1998) 3016; E.M. Lipmanov, hep-ph/9806442 V. Barger, hep-ph/9808353; S.M. Bilenky, C. Giunti and W. Grimus, hep-ph/9809368; S.M. Bilenky, C. Giunti, W. Grimus and T. Schwetz, hep-ph/9809466. 
[10] B. Brahmachari and R.N. Mohapatra, hep-ph/9805429 R.N. Mohapatra, hep-ph/9808236.

[11] LSND Collaboration, C. Athanassopoulos et al., Phys. Rev. Lett. 75 (1995) 2650; ibid. 77 (1996) 3082; ibid. 81 (1998) 1774.

[12] The LSND results will be tested by the KARMEN experiment, talk by B. Armbruster at 33rd Rencontres de Moriond : Electroweak Interactions and Unified Theories, Les Arcs, France, March 1998, and talk by B. Zeitnitz at 'Neutrino 98', Takayama, Japan, June 1998, and also by the BooNE experiment, E. Church et al., nucl-ex/9706011.

[13] C. Caso et al., Eur. Phys. J. C 3 (1998) 1.

[14] N. Cabibbo, Phys. Rev. Lett. 10 (1964) 531; M. Kobayashi and T. Maskawa, Prog. Theor. Phys. 49 (1973) 652. 


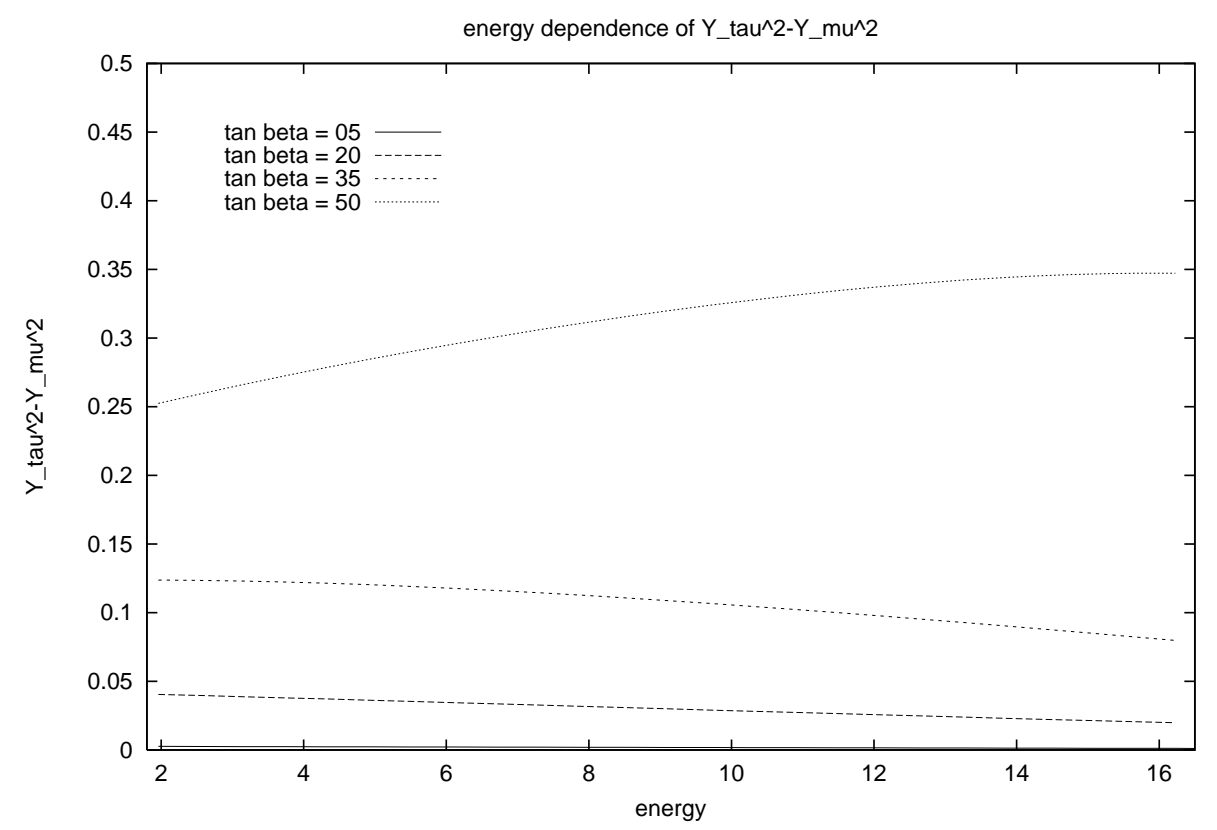

Figure 1: The energy dependence of the values of $y_{\tau}^{2}-y_{\mu}^{2}$ with various values of $\tan \beta$. These values are the coefficients of the RGEs of $\kappa_{r}$ and $\sin ^{2} 2 \theta_{23}$. 


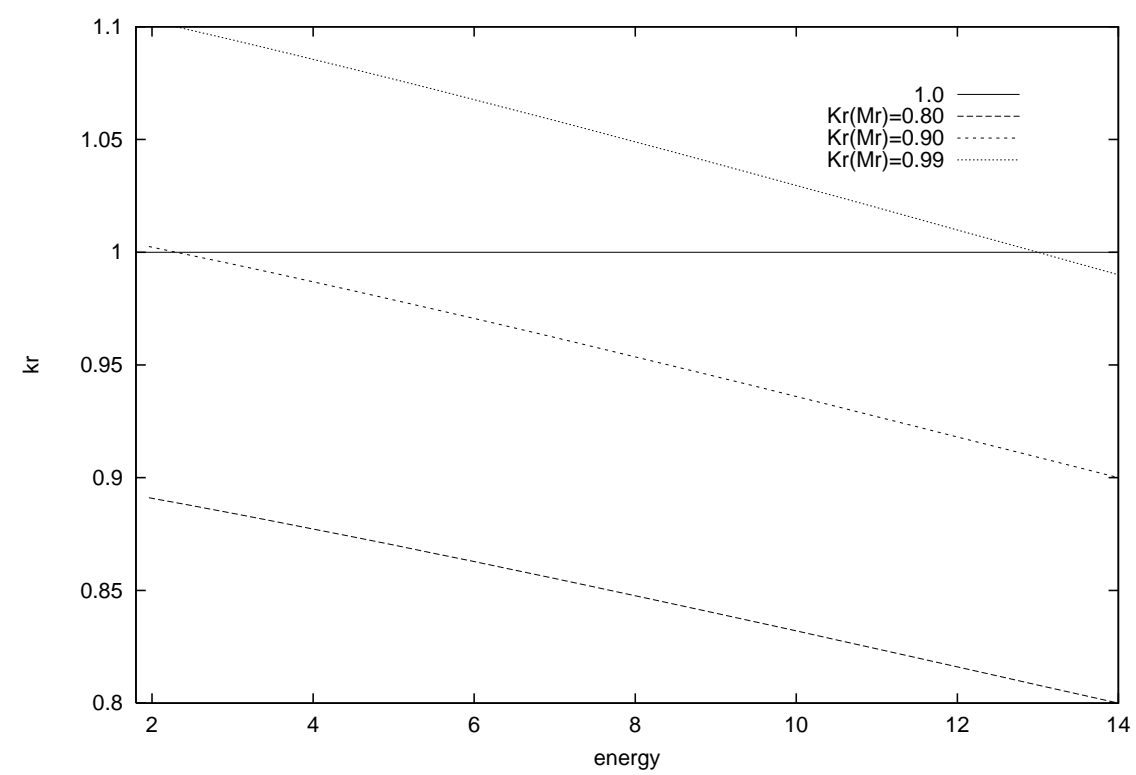

Figure 2(a): $\mathcal{M}_{\mathcal{R}}=10^{14} \mathrm{GeV}$

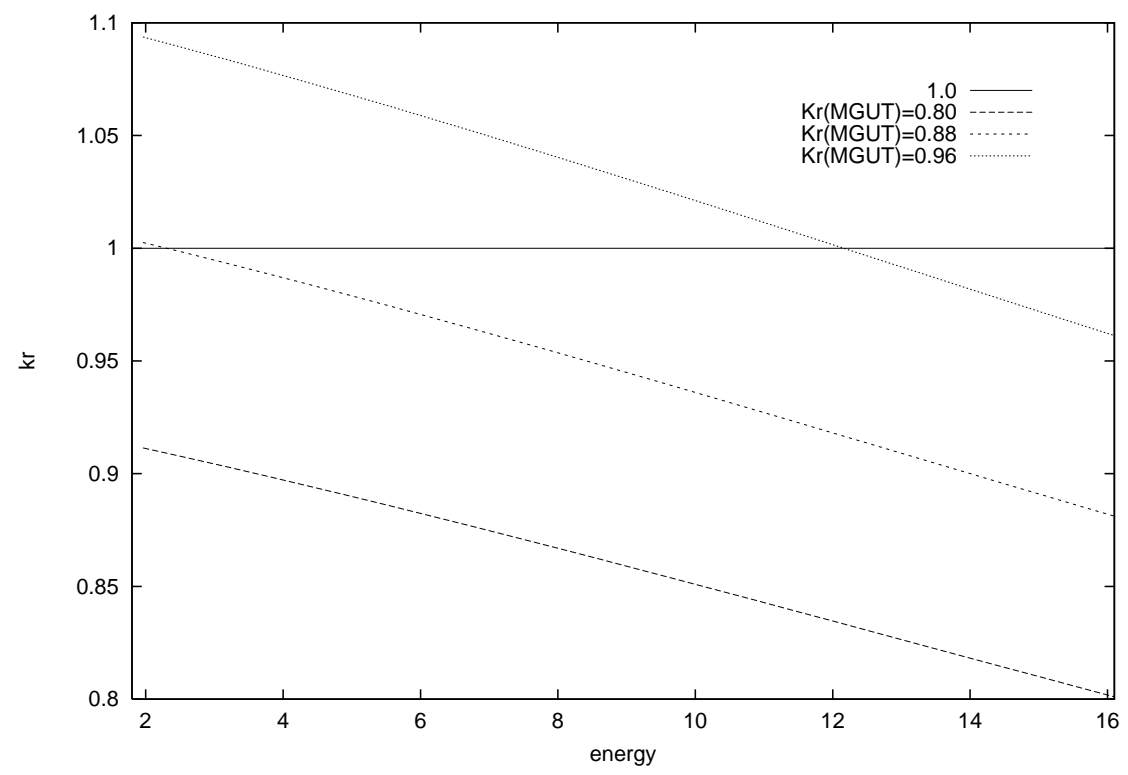

Figure 2(b): $\mathcal{M}_{\mathcal{R}}=10^{16} \mathrm{GeV}$

Figure 2: The energy dependence of $\kappa_{r}$ with $\tan \beta=50$. (a): The initial condition is fixed as $\kappa_{r}\left(\mathcal{M}_{\mathcal{R}}\right)=0.8,0.9$, and 0.99 at $\mathcal{M}_{\mathcal{R}}=10^{14} \mathrm{GeV}$. (b): The initial condition is fixed as $\kappa_{r}\left(\mathcal{M}_{\mathcal{R}}\right)=0.8,0.88$, and 0.96 at $\mathcal{M}_{\mathcal{R}}=10^{16} \mathrm{GeV}$. 


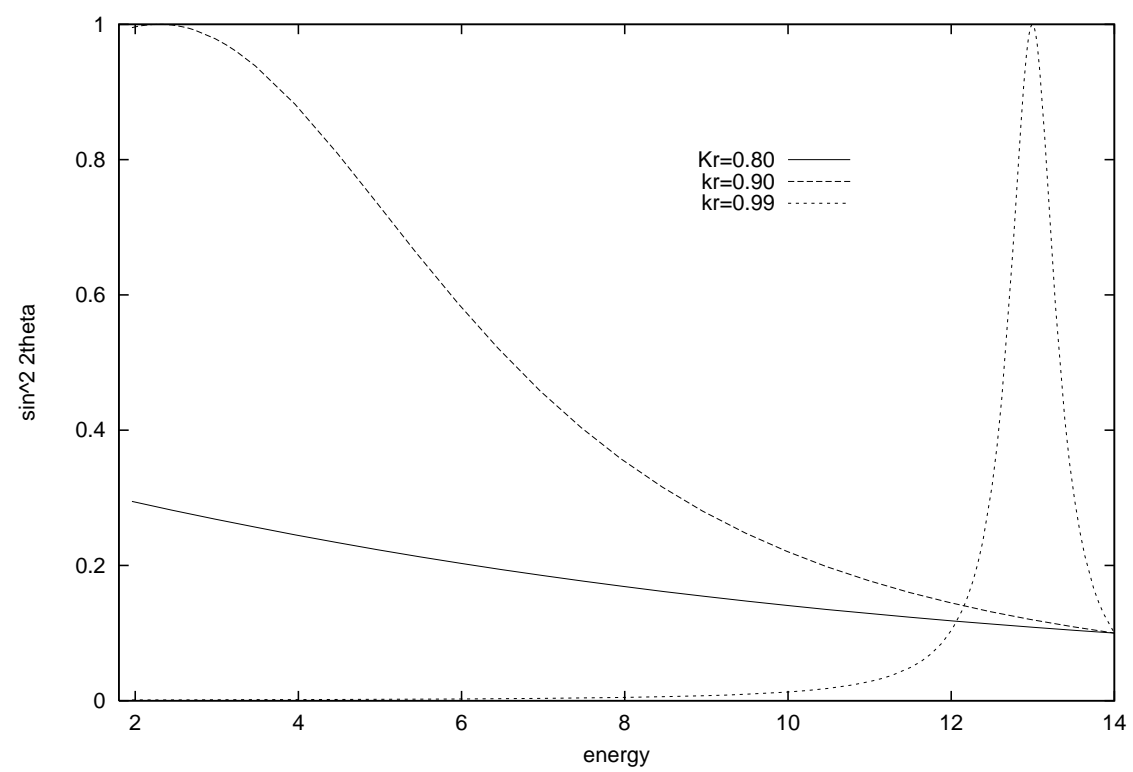

Figure 3(a): $\mathcal{M}_{\mathcal{R}}=10^{14} \mathrm{GeV}$

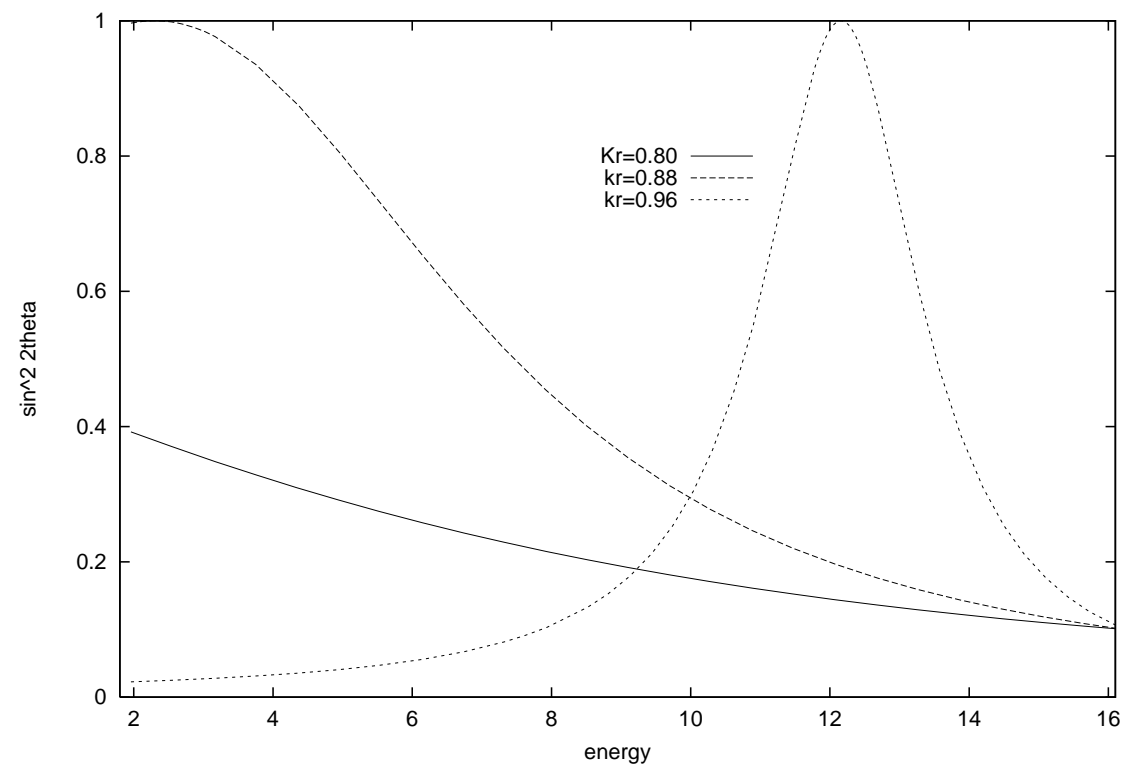

Figure 3 (b): $\mathcal{M}_{\mathcal{R}}=10^{16} \mathrm{GeV}$

Figure 3: The energy dependence of $\sin ^{2} 2 \theta$ with $\tan \beta=50$. We show two cases of (a): $\mathcal{M}_{\mathcal{R}}=10^{14} \mathrm{GeV}$ and (b): $10^{16} \mathrm{GeV}$. 


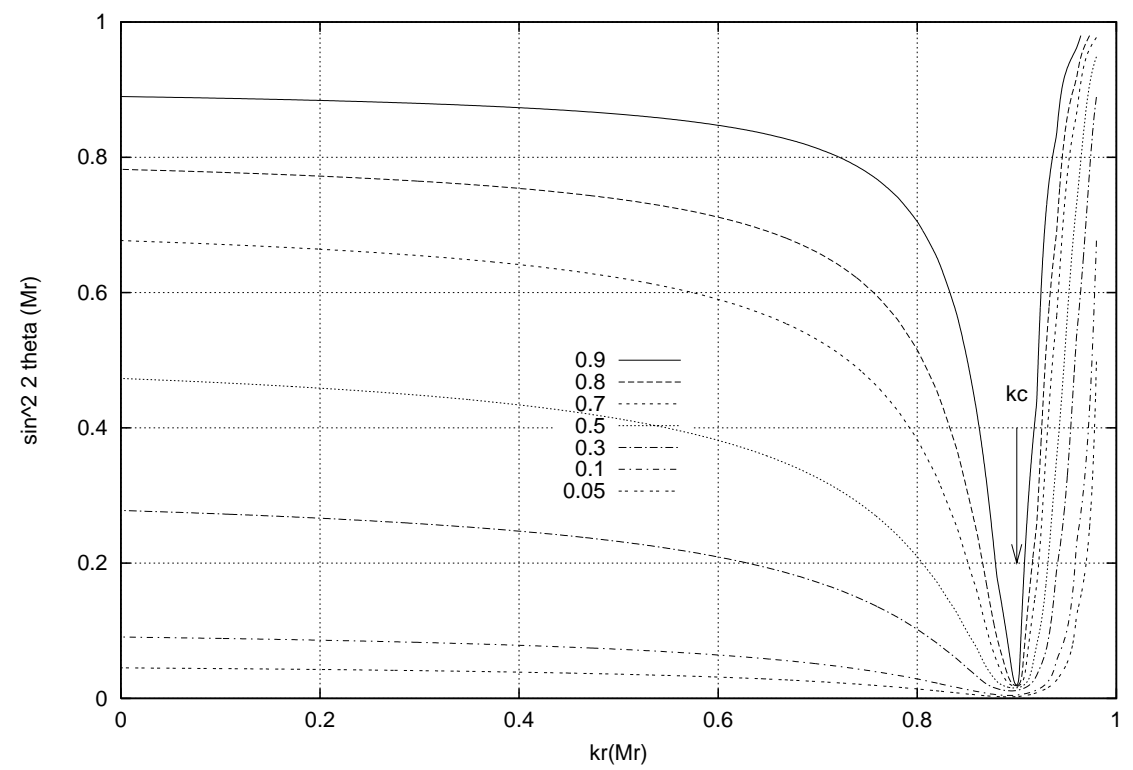

Figure $4(\mathrm{a}): \tan \beta=50, \mathcal{M}_{\mathcal{R}}=10^{14} \mathrm{GeV}$

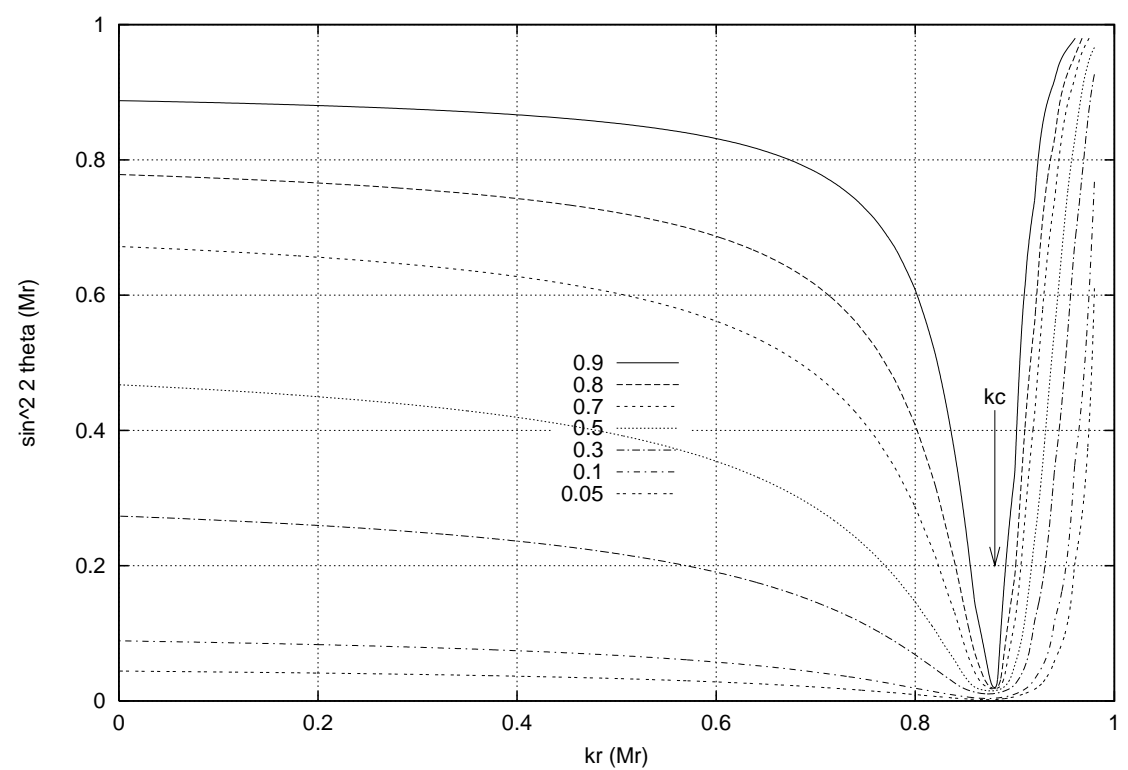

Figure 4(b): $\tan \beta=50, \mathcal{M}_{\mathcal{R}}=10^{16} \mathrm{GeV}$

Figure 4: The contour plots of the mixing angle $\sin ^{2} 2 \theta_{23}$ at the weak scale with $\tan \beta=50$. We show two cases of $(\mathrm{a}): \mathcal{M}_{\mathcal{R}}=10^{14} \mathrm{GeV}$ and (b):10 ${ }^{16} \mathrm{GeV}$. The horizontal axes show the value of $\kappa_{r}$ at $\mu=\mathcal{M}_{\mathcal{R}}$ scale, and the vertical axes show the mixing angle at $\mu=\mathcal{M}_{\mathcal{R}}$ The values of $\sin ^{2} 2 \theta_{23}$ at the weak scale are determined by inputting the initial values of $\sin ^{2} 2 \theta_{23}\left(\mathcal{M}_{\mathcal{R}}\right)$ and $\kappa_{r}\left(\mathcal{M}_{\mathcal{R}}\right)$. Around $\kappa_{c}$, the RGE effects make the weak scale mixing be large at any initial conditions. 


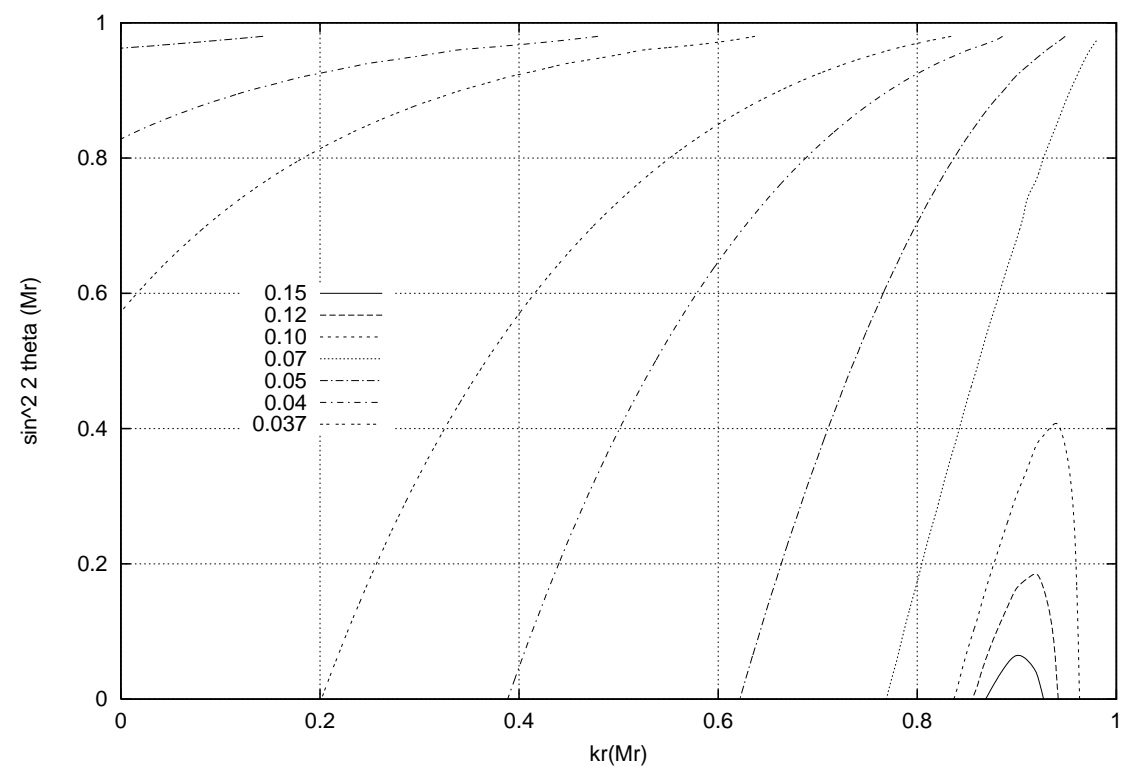

Figure 5(a): $\tan \beta=50, \mathcal{M}_{\mathcal{R}}=10^{14} \mathrm{GeV}$

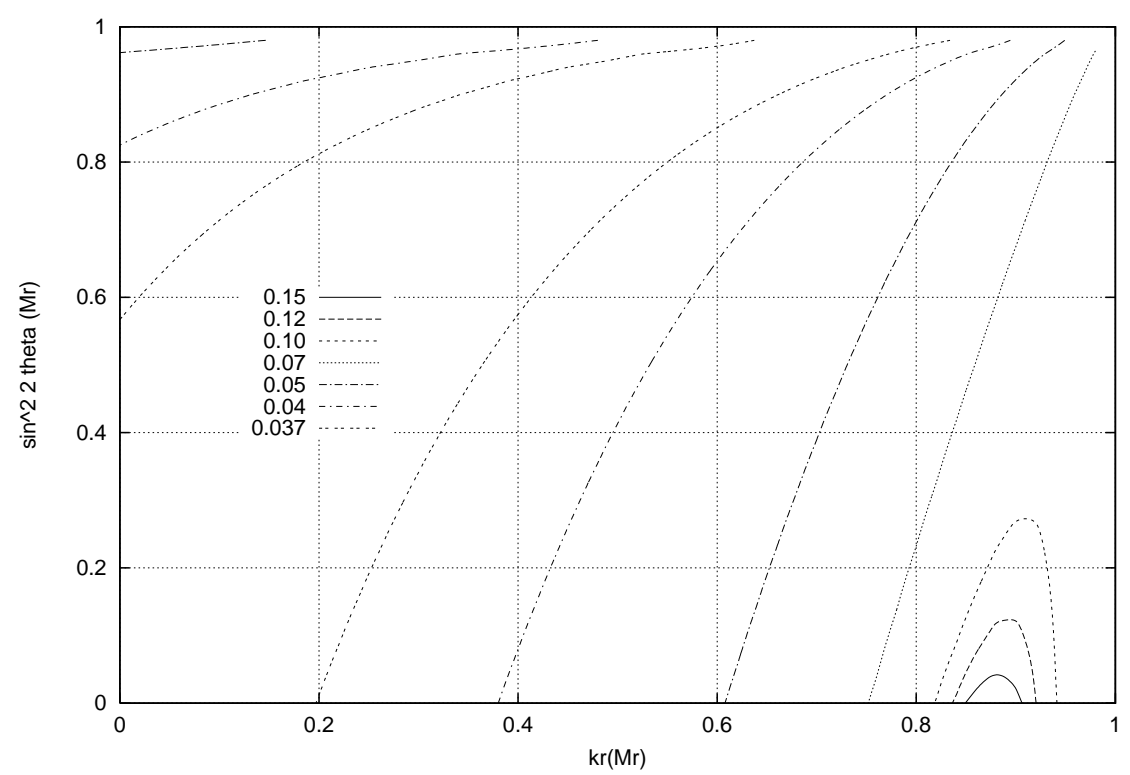

Figure 5(b): $\tan \beta=50, \mathcal{M}_{\mathcal{R}}=10^{16} \mathrm{GeV}$

Figure 5: The contour plots of the heaviest neutrino mass $m_{3}$ at the weak scale with $\tan \beta=50$. The horizontal and vertical axes are the same as Figs. 4 . The values of $m_{3}$ at the weak scale are determined by inputting the initial values of $\sin ^{2} 2 \theta_{23}\left(\mathcal{M}_{\mathcal{R}}\right)$ and $\kappa_{r}\left(\mathcal{M}_{\mathcal{R}}\right)$, and the experimental value of $\delta m_{23}^{2}=1.3 \times 10^{-3} \mathrm{eV}^{2}$. As the mass becomes heavy, the region of $\kappa_{r}\left(\mathcal{M}_{\mathcal{R}}\right)$ is limited around $\kappa_{c}$. 


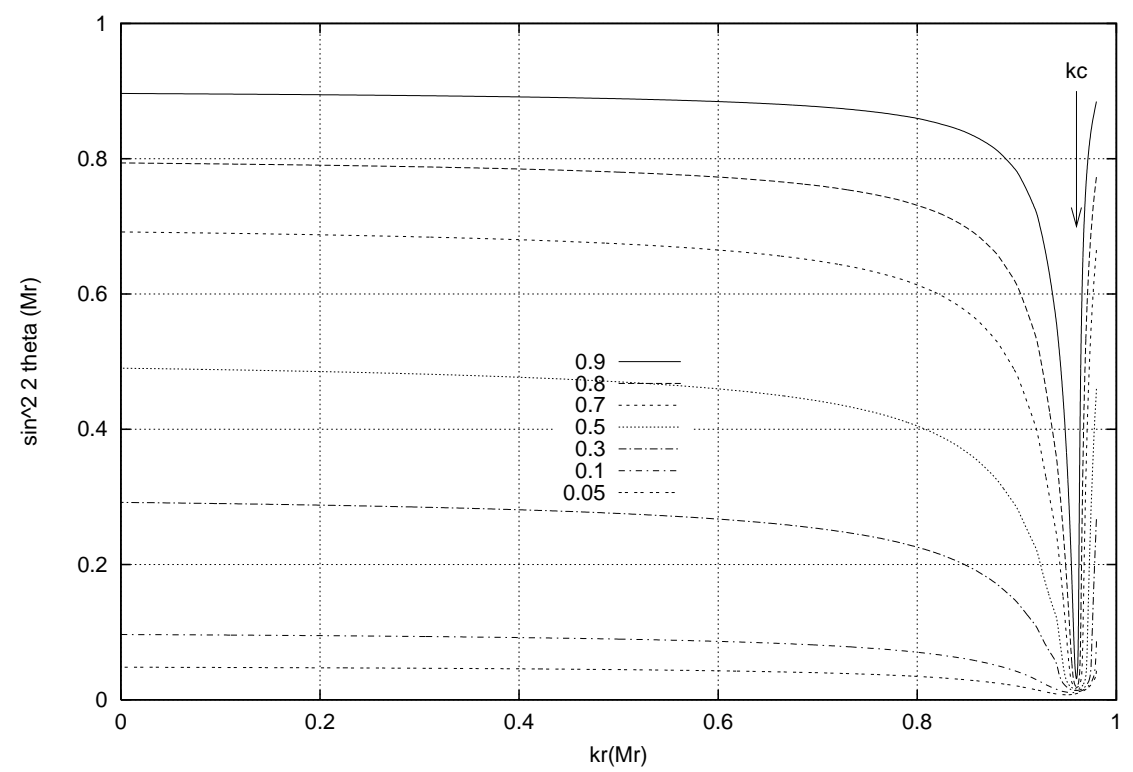

Figure 6 (a): $\tan \beta=35, \mathcal{M}_{\mathcal{R}}=10^{14} \mathrm{GeV}$

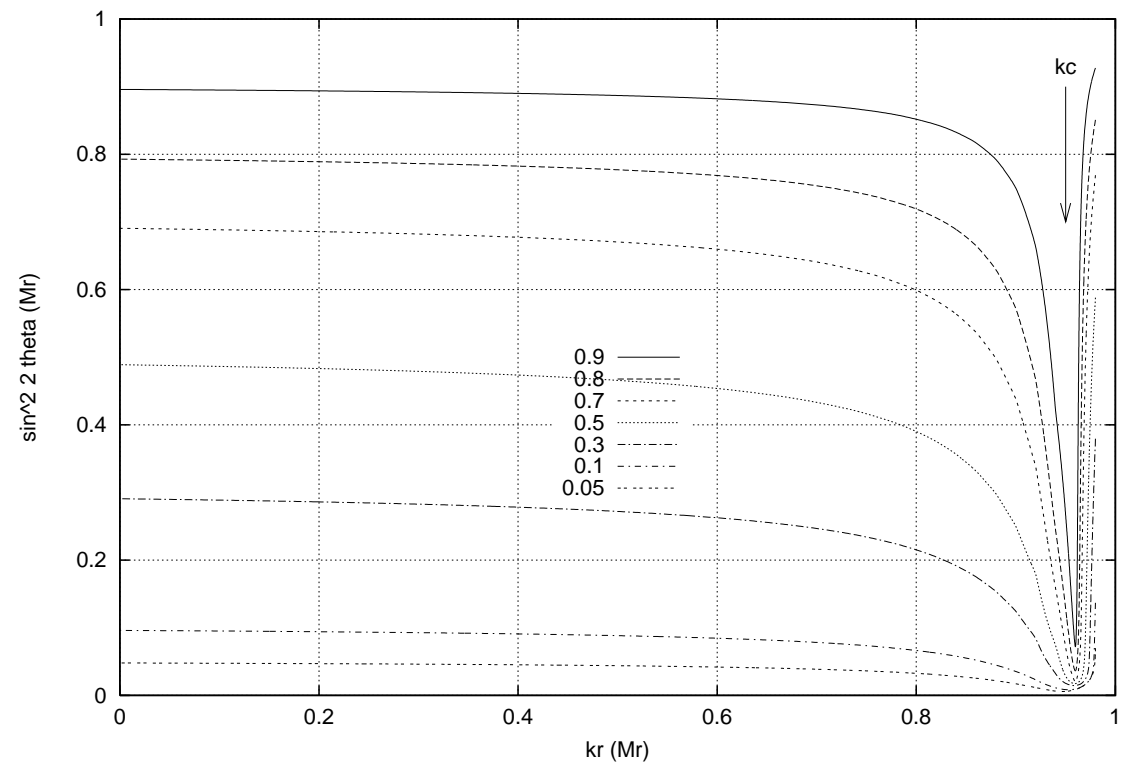

Figure [6(b): $\tan \beta=35, \mathcal{M}_{\mathcal{R}}=10^{16} \mathrm{GeV}$

Figure 6: The contour plots of the mixing angle $\sin ^{2} 2 \theta_{23}$ at the weak scale with $\tan \beta=35$. In this case $\kappa_{c}$ is larger than that of Figs. 4. 


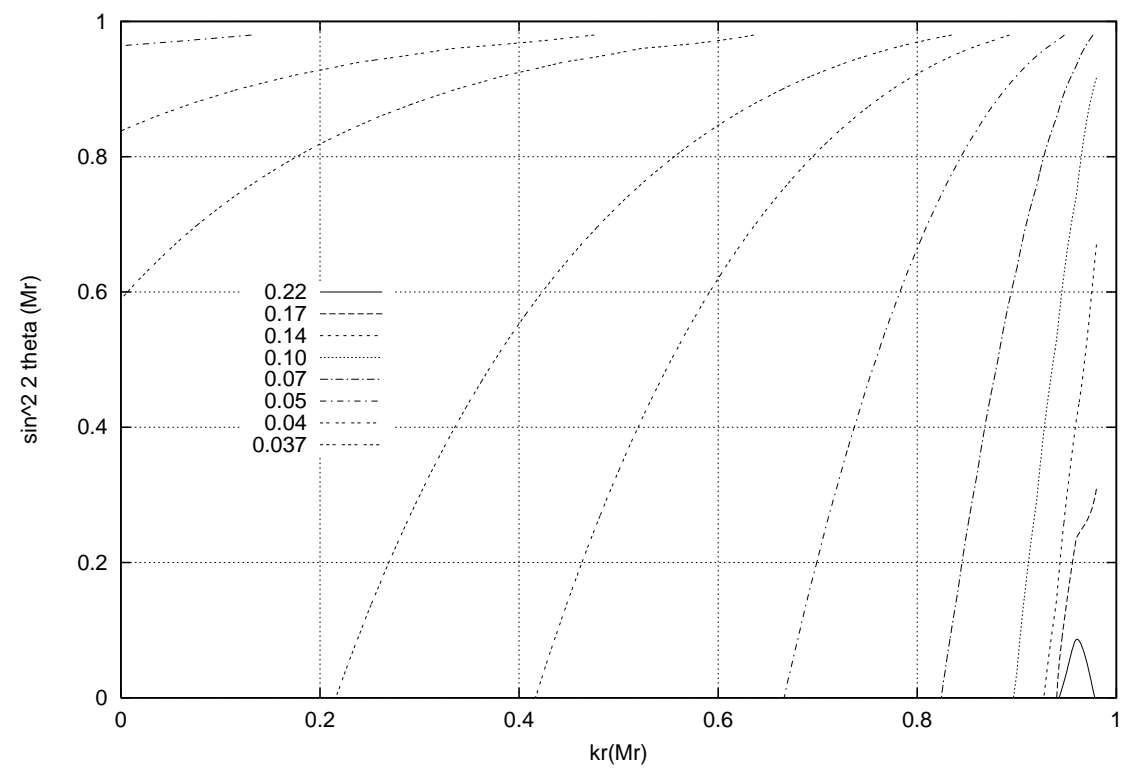

Figure 7(a): $\tan \beta=35, \mathcal{M}_{\mathcal{R}}=10^{14} \mathrm{GeV}$

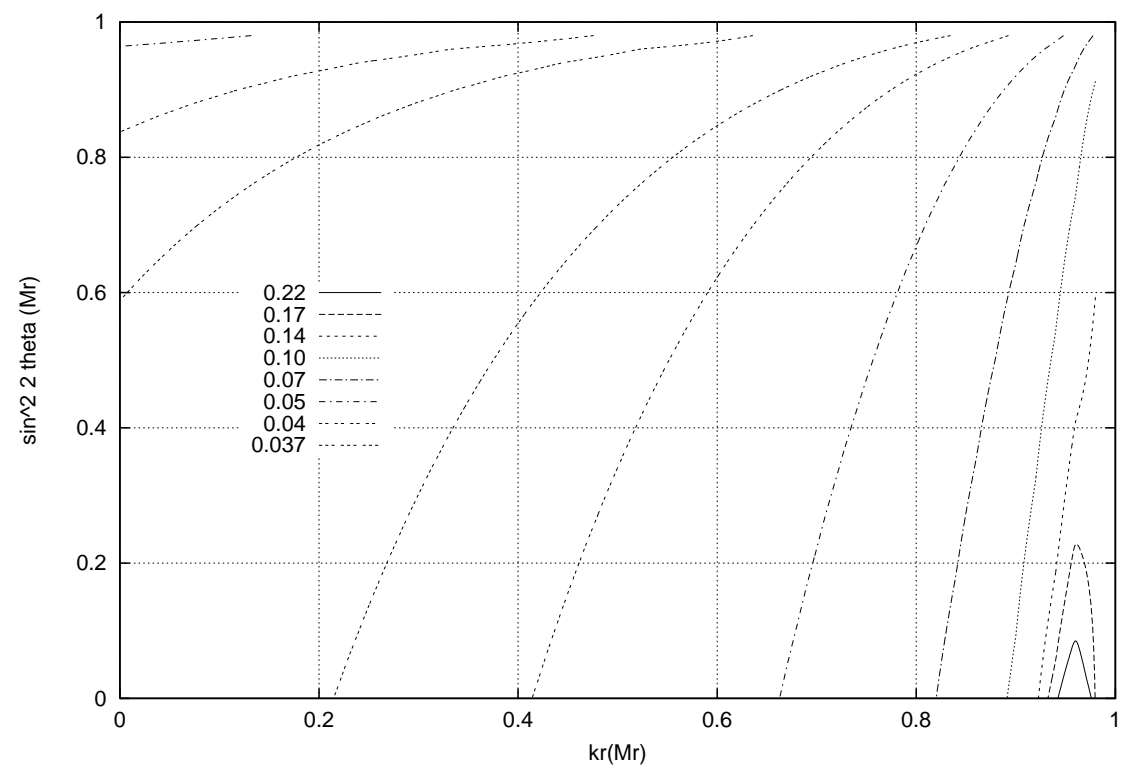

Figure 7(b): $\tan \beta=35, \mathcal{M}_{\mathcal{R}}=10^{16} \mathrm{GeV}$

Figure 7: The contour plots of the heaviest neutrino mass at the weak scale with $\tan \beta=35$. The region where $m_{3}$ is larger than $O(0.1 \mathrm{eV})$ corresponds to the region in which the maximal mixing angle is realized at low energy. 